\title{
Formation and dissolution of leaky clusters
}

\author{
S. Pfalzner
}

Max-Planck-Institut für Radioastronomie, Auf dem Hügel 69, 53121 Bonn, Germany

e-mail: spfalzner@mpifr-bonn.mpg.de

Received 28 July 2011 / Accepted 12 October 2011

\section{ABSTRACT}

\begin{abstract}
Context. Massive Galactic clusters $\left(>1000 M_{\odot}\right.$ ) exhibit a clear correlation between cluster density, size, and age and can be sorted into two categories, i.e. starburst and leaky clusters. The reason for having two types of massive clusters is still an open question; however, the answer is probably connected to the different formation histories of the two types.

Aims. In this study we concentrate on leaky clusters and investigate possible formation scenarios and the gas expulsion phase. Methods. We use the existing observational data and numerical results of embedded cluster properties.

Results. Assuming that a clear correlation between cluster density, size, and age exists, it is shown that the density-radius development over time for embedded clusters can be approximated by $\rho \approx 100 * r^{-1.3} M_{\odot} \mathrm{pc}^{-3}$. The consequences for the star formation process in leaky clusters are discussed and found to favour an inside-out star formation scenario with an initially low, but later accelerated, star formation rate. It is shown how the leaky clusters form in a unique, sequential manner and how rapid gas expulsion is responsible for the $80-90 \%$ mass loss over the next 20 Myr.
\end{abstract}

Key words. open clusters and associations: general - stars: formation

\section{Introduction}

Most stars form in dense clusters embedded within giant molecular cloud clumps (Lada \& Lada 2003; Pudritz 2002). Given the relatively high number of clusters younger than 10 Myr compared to the much lower number of older clusters in equivalent age spans, the majority of clusters must rapidly disolve soon after their formation. As no clear correlation between cluster mass and age has been found, until recently the general view has been that in the Milky Way, star formation results in a multitude of clusters with hardly any constraints on stellar content, size, or density. However, this view had to be revised at least for clusters more massive than $1000 M_{\odot}$. Pfalzner (2009) showed that these massive clusters follow two separate sequences. Although these are difficult to distinguish in the mass-age plane they immediately become apparent in density-radius space. In both cases the expulsion of gas from the cluster via stellar winds, ionisation and supernovae explosions(Hills 1980; Goodwin \& Bastian 2006; Bastian et al. 2008; Baumgardt \& Kroupa 2007) leads to an expansion (see Fig. 1 here and more details in Pfalzner 2009, Fig. 2).

The reason for this bi-modal development is currently not understood. One might think that a difference in cluster mass might cause it. However, Fig. 1 shows that at an early age $(<4 \mathrm{Myr})$ both types of clusters have nearly the same mass, but the cluster radii in the two groups (diamonds) differ by more than a factor 10. An additional difference is that the mass of starburst clusters remains more or less constant, whereas that of leaky clusters reduces to about $10 \%$ to $20 \%$ of its initial value. A hint of the origin of the two groups of massive clusters is the obvious difference in the location of the clusters - all starburst clusters are located in regions of high stellar density - near the galactic centre or in the spiral arms - whereas the leaky clusters are situated in lower density areas. Clearly the answer to why massive clusters exist in two categories must lie in their formation

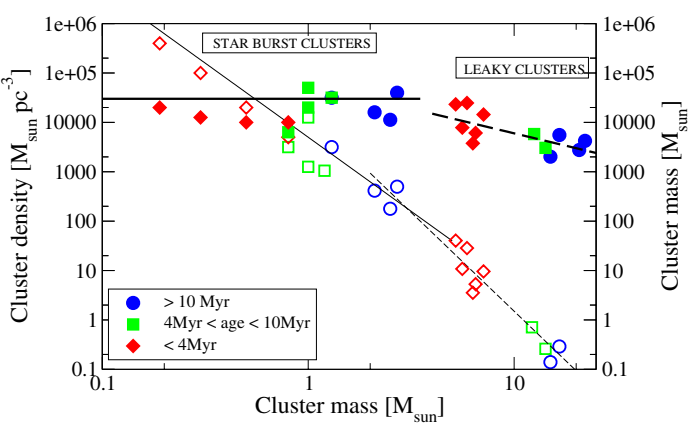

Fig. 1. Cluster mass as a function of cluster size (full symbols) for exposed clusters more massive than $10^{3} M_{\odot}$. The diamond symbols represent clusters younger than $4 \mathrm{Myr}$, the squares clusters in the age range $4 \mathrm{Myr}<t_{\mathrm{c}}<10 \mathrm{Myr}$, and the circles clusters with ages $10 \mathrm{Myr}<t_{\mathrm{c}}<$ 20 Myr. The thick line shows that the cluster mass remains more or less constant for the starburst cluster sequence, whereas the leaky cluster mass rapidly declines with cluster age (thick dashed line). The values were taken from Figer (2008), Wolff et al. (2007), and Borissova et al. (2008). In addition, the relation between the cluster density and cluster radius are shown by empty symbols as in Pfalzner (2009).

process. In this paper we concentrate on the formation and development of leaky clusters which start out with masses of the order of $10^{4} M_{\odot}$ and loose $80-90 \%$ of this mass over the next 20 Myr after gas expulsion.

These clusters form as a bound entity that dissolves to a large degree after gas expulsion. The emphasis of this study lies on the formation process of leaky clusters. The precursors to leaky clusters must be found among the still embedded clusters. But the question is which embedded clusters develop into leaky clusters and how does this development proceed.

One possibilty would be that leaky clusters form by merging of sub-clusters as recently suggested by several authors for less massive cluster (see, for example, Küpper et al. 2011, and 
references therein). However, to form a leaky cluster this would require that several clusters like the Orion nebula cluster (ONC) with $>1000$ stars or hundreds of small-N clusters form in close vicinity and merge within $<1$ Myr. So far there are no observational indications of such a formation process for leaky clusters. Here we stick to the more conservative picture of a leaky cluster forming as a single entity.

In the following, the existing data on embedded cluster properties are used to constrain the conditions of leaky cluster formation and the gas expulsion phase. The idea is that a better understanding of the formation process and later development of leaky clusters will eventually answer the open question of why leaky and starburst clusters form and develop differently.

\section{Method}

This work uses available observational data of the properties of embedded cluster by Lada \& Lada (2003) to propose a possible formation history for leaky clusters. In principle one could deduce the star formation history of massive clusters by looking at the age distribution within single clusters. However, there are two problems with this approach: i) leaky clusters show considerable mass loss within the first 10 Myr of their development, and ii) it is not clear how long the process of star formation actually takes in such clusters - it could be coeval or take up to $\sim 10 \mathrm{Myr}$.

In young embedded clusters like the ONC, wide spreads in colour-magnitude diagrams (CMD) have been observed, which have previously been interpreted as age spreads of up to $10 \mathrm{Myr}$ in association members (Palla \& Stahler 2000). However, other authors have suggested that the observed CMD spreads are due to a mixture of photometric errors, variability in pre-MS stars (Herbst et al. 1994), binarity (Preibisch \& Zinnecker 1999), episodic accretion phases (Baraffe et al. 2009), or a spread in line-of-sight distances or extinctions. Currently age spreads of 1 to $3 \mathrm{Myr}$ are regarded as realistic for the $\mathrm{ONC}$. However, the age of Cyg OB2 was originally estimated as 1-3 Myr when only considering OB stars. A concentration of 5 to 7 Myr old A stars was found just to the south of Cyg OB2 (Drew et al. 2008), while an unclustered spread of evolved stars up to $10 \mathrm{Myr}$ old was identified over a wider area (Comerón et al. 2008). Wright et al. (2010) favour the view that the present-day OB stars are products of the latest phase of star formation, but they reason that at least one more epoch of star formation took place beforehand. In summary, the observations of the stellar ages in single star clusters give a rather unclear picture of the duration and the dynamics of the star formation process.

Rather than looking at the star formation history in one single cluster, one could look at clusters of different ages and construct a star formation history from this sequence. Mature leaky clusters consist of thousands of stars, but nevertheless they must have started out in the embedded phase containing just a few dozen stars, thus acquiring additional members via star formation. The straightforward approach would be to determine the ages of the embedded clusters and sort them temporally to obtain the formation history of leaky clusters. Unfortunately, this is hindered because age determination in the embedded phase is even more complicated than in the early exposed phases owing to the high uncertainty of the pre-main sequence (PMS) stellar evolution models. Although embedded clusters are usually attributed ages in the 0 to $2 \mathrm{Myr}$ range, the error bar is roughly the same. As a consequence, ordering embedded clusters according to their age would be extremely prone to error.
For the exposed phase, an important consequence of the sequential nature of the leaky cluster development in the cluster radius-age space is that the radius of a cluster can be used to determine its age (see Fig. 3 in Pfalzner 2009). For leaky clusters a fit to the observational data gives a correlation between the average cluster radius $r_{\mathrm{cl}}$ and its age $t_{\mathrm{cl}}$ of the form:

$$
t_{\mathrm{cl}} / \mathrm{Myr} \sim 0.15\left(r_{\mathrm{cl}} / \mathrm{pc}\right)^{3 / 2} \text {. }
$$

One way out of the dilemma of determining a formation history for leaky clusters is the assumption that, similar to the exposed phase of the leaky cluster development, a similar correlation exists between the cluster radius and its age in the embedded phase. In other words, we postulate a relation between cluster membership, i.e. of the number of stars already formed, and age in the embedded phase for star clusters that eventually develop into leaky clusters. This is the simplest assumption we can make, and it implies that star formation takes place in these clusters.

Now the same underlying reasoning as for exposed clusters is used implicitly: since each of the exposed leaky clusters has the same developmental history, it seems reasonable to assume that their precursors in the embedded phase also have a common history. However, not every embedded cluster will develop into a massive leaky cluster. Only if there is enough matter in the surrounding cloud is this developmental path open. Clusters with too low mass content in their surrounding stop forming stars as soon as they run out of material, well before they have reached masses in excess of $10^{3} M_{\odot}$. Most likely these starved clusters quickly disperse so that their surface density drops below the detection line more or less immediately.

To investigate the consequences of the assumption of a common history of the precursors of the leaky clusters, we use the data of embedded cluster properties by Lada \& Lada (2003). They selected these clusters within $2 \mathrm{kpc}$ of the Sun in such a way that the group must have a high enough stellar-mass volume density to render it stable against tidal disruption by the Galaxy (i.e., $\rho>0.1 M_{\odot} \mathrm{pc}^{-3}$ ) and by passing interstellar clouds (i.e., $\rho>1 M_{\odot} \mathrm{pc}^{-3}$ ). In addition, the group should contain enough members to ensure that its evaporation time is longer than the typical lifetime of Galactic open clusters. It means that existing clusters with densities $<5 M_{\odot}\left(r_{\mathrm{cl}} / \mathrm{pc}\right)^{-3}$ are possibly not included in this sample given in (Lada \& Lada 2003, see dashed line in Fig. 2). In addition clusters with densities $<31.5 M_{\odot} \mathrm{pc}^{-2}\left(r_{\mathrm{cl}} / \mathrm{pc}\right)^{-1}$ cannot be detected against the field of background stars.

Carpenter et al. (2000) state that for their sample the observational density limits imply that clusters of density $<31.5 M_{\odot} \mathrm{pc}^{-2}\left(r_{\mathrm{cl}} / \mathrm{pc}\right)^{-1}$ are too tenuous to be detected against the background of the field. If this limit also applies to the Lada \& Lada (2003) data sample (see dashed line in Fig. 3), the absence of lower density embedded clusters might be an artifact of observational limitations. On the other hand, this limit might also be too stringent and the exposed leaky clusters with sizes $>10 \mathrm{pc}$ can be detected despite their density being relatively low compared to the background density.

\section{Results}

\subsection{Density development in the embedded phase}

Even the most massive leaky cluster must have started out at some point with few stars. Figure 2 shows the lower part of Fig. 1, the leaky cluster sequence, but augmented by the data sample of embedded clusters from Lada \& Lada (2003) with 


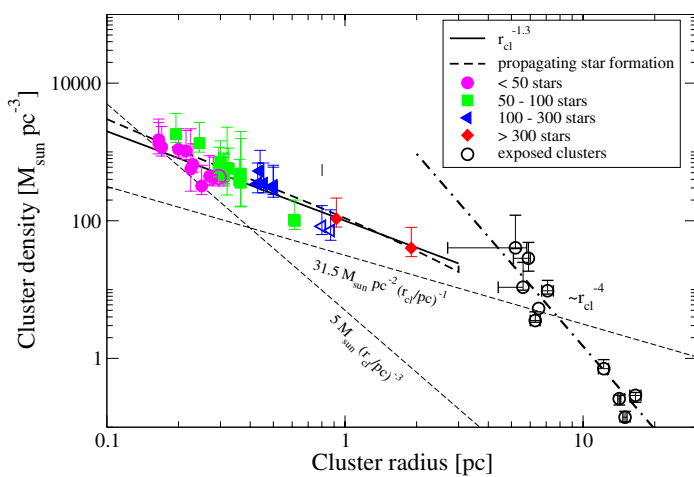

Fig. 2. Cluster density as a function of cluster size for the embedded clusters listed in Lada \& Lada (2003) (coloured symbols) and the leaky clusters from Pfalzner (2009) (black symbols). The open coloured symbols for the embedded clusters indicate the values for IRAS 06155+2319, GEM1, NGC 2281 and GEM4.

known sizes and masses. Because this data set is limited to embedded clusters within $2 \mathrm{kpc}$ from the Sun, its members are far enough away from the Galactic centre and the spiral arms, so it is most likely free of precursors to starburst clusters. Figure 2 implies that the precursors of leaky clusters form an extension of the leaky cluster sequence towards smaller radii $(<3 \mathrm{pc})$ at density of $\geq 100 M_{\odot} \mathrm{pc}^{-3}$. However, the embedded clusters do not form a direct continuation of the slope of the main evolutionary sequence to lower radii, but the density development is flatter. This is because expansion due to gas expulsion has not started yet, and star formation is still going on.

In Figs. 2 and 4 clusters with $<50,50-100,100-300$ and $>300$ members have been assigned to four different groups via distinct colours. The average radius of these cluster groups increases with the number of members. Following the timesequence hypothesis - that age and radius are also correlated in the embedded phase - we may interpret Fig. 2 as follows. A cluster starts with an initial phase where a limited number of stars form $(<100)$, the cluster radius increases by a factor $2-3$, and the cluster becomes more diffuse. The relatively high number of embedded clusters is indicative of the slow star formation rate at this stage. Afterwards the star formation rate increases considerably and the cluster radius nearly grows by a factor of 10 . During this phase the cluster density stays nearly constant or decreases only slightly. Whether these are actually two distinct phases or a continuous transition cannot be decided based on current data. However, considerable acceleration of star formation occurs at a cluster size of about $0.7-1 \mathrm{pc}$ when more than 100 members are formed. In Fig. 2 the dependency of the cluster density $\rho$ on the cluster radius $r_{\mathrm{cl}}$

$\rho \approx 100 r_{\mathrm{cl}}^{-1.3}$

provides only a rough clue to the development because the original data set not specified but presumably large errors in cluster radius do not justify a proper fit at this point. The error in mass has been estimated as approximately a factor 2 by Lada \& Lada (2003), this error was taken in Fig. 2 for determining the stellar densities.

Figure 2 shows that the average density in low $N$-clusters which in the picture presented here corresponds to the youngest clusters - is often more than ten times higher than that of the ONC. This means that nearly all stars are exposed to interactions with neighbouring young forming stars in these early phases, which most likely influences star and planet formation processes. Properties that might be altered by this high stellar

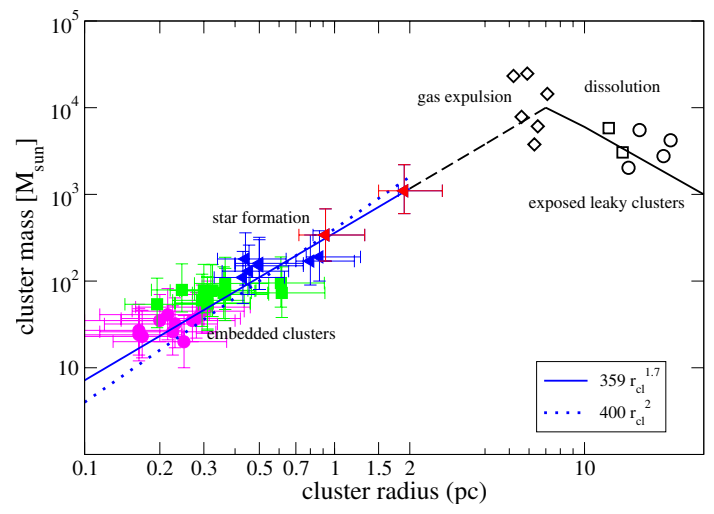

Fig. 3. Mass as a function of radius for embedded clusters. The data points are taken from Lada \& Lada (2003), and the best fit for $a R^{b}$ gives $a=359 \pm 15$ and $b=1.71 \pm 0.07$. The symbols are the same as depicted in Fig. 2.

density are, for example, the binary frequency, the density profile in the circumstellar discs, etc. Although clusters like the ONC show a similar density in the central cluster regions to that of low- $N$ clusters, most stars are located in areas where the environment is less important.

The relative importance of these environmental effects on star and planet formation is still an open question, however, this knowledge of the density development during the star formation process will allow us to determine the encounter probability in such clusters better in future investigations. In the future this can show in how far the high stellar density influences star and planet formation processes in leaky cluster environments.

As mentioned before, not all embedded clusters included in Fig. 2 will necessarily develop into leaky clusters: although the Lada \& Lada (2003) sample probably excludes precursors to starburst clusters, it does not exclude precursors of lower mass clusters. Only if there is sufficient material in the surrounding cloud will the cluster continue to evolve towards the leaky cluster sequence. It is, for example, unlikely that IC 348 at the leftward end of the embedded track in Fig. 2 will develop into a leaky cluster because its gas content is too low. For embedded clusters that develop into lower mass exposed clusters, the relation of Eq. (2) probably serves as an upper density limit.

However, clusters that contain sufficient gas will eventually join up with the leaky cluster sequence when the gas expulsion has caused an expansion to $\sim 2-4 \mathrm{pc}$. It is most likely that only embedded clusters as dense as those shown in Fig. 2 can be the percursors of the $<4$ Myr old leaky clusters. Lower density clusters might exist, but those that are precursors to leaky clusters will be at the high density limit (for a given radius) of this data set.

In Fig. 2 the dependence of the cluster density on the cluster radius could also be interpreted as an observational selection artefact, in the sense that many clusters show multiple peaks in their density. If the low-mass clusters all represented one such core and the higher mass clusters several cores with low density regions in between, one could obtain a similar diagram. However, Fig. 3 shows clearly that it is really a mass-radius relation.

By plotting the cluster mass of the Lada \& Lada (2003) data as a function of the cluster radius (see Fig. 3), a relation of the form

$M_{\mathrm{c}}=a r_{\mathrm{cl}}^{b}$ 
with $a=359 \pm 15$ and $b=1.71 \pm 0.07$ is obtained. If the cluster mass is corrected to a lower mean stellar mass, this would change to $a R^{b}$ with $a=451 \pm 15$ and $b=1.71 \pm 0.07$, however, given the large errors, an $R^{2}$-relation could also be considered. It can be seen that the extension of Eq. (3) to larger cluster radii or the equivalent at later times in the cluster development meets with the leaky cluster track at the point where these clusters become exposed. Figure 3 therefore represents the formation, gas expulsion and dissolution phase of the leaky cluster sequence.

In the following a rough estimate of the errors in cluster radii is attempted. There are several sources that could lead to such an error. The embedded nature means that not all stars are detected. If mass segregation is primordial, the higher likelihood of lowmass stars to be missed could lead to too small a detected radius. In addition, many of the embedded clusters are not spherically symmetric but are more extended in one direction. In Fig. 3 it was assumed that the error in cluster radius is approximately half its actual value.

Adams et al. (2006) note a related correlation between cluster radius and number of stars $N$, namely, $r_{\mathrm{cl}} \propto=N^{1 / 2}$. However, they interpret this correlation as a static property of embedded clusters and not as an outcome of temporal development of the cluster formation process. Nevertheless, with $M \propto N$ it would follow that $M_{\mathrm{c}} \propto r_{\mathrm{cl}}^{2}$, which could also fit the data considering the errors (see Fig. 3).

If the trend expressed by Eq. (3) is not an evolutionary sequence at all, then one has to explain why only certain massradius combinations seem to exist for young embedded clusters. Selection effects could play a role, so it would be hard to detect clusters with $M_{\mathrm{c}}<100 M_{\odot}$ and $r_{\mathrm{cl}}<2$ pc. However, it would be unlikely to miss clusters with, for example, $M_{\mathrm{c}}>500 M_{\odot}$ and $r_{\mathrm{cl}} \sim 0.5 \mathrm{pc}$, which are clearly not present in the sample. One way out would be a mass-size relation for cores (see, for example, Swift \& Williams 2008). However, then the percursors of the clusters would all have to form from thinly spread clusters with very low initial surface densities and be all below the detection limit. These clusters would then form stars within a more or less fixed radius finally reaching such a density that they can be detected. This explanation works very well for low-mass clusters. However, in such a picture the precursors of the leaky clusters should be embedded clusters with cloud masses $M_{\text {cloud }}>1000 M_{\odot}$ containing thousands of stars within $r_{\mathrm{cl}} \sim 3-6 \mathrm{pc}$. Such clusters should be detectable in the solar neighbourhood. As this is not the case, the only explanation would be that they are either rare or very short-lived.

We therefore prefer here the model of interpreting the massradius relation as a time sequence. In this model the existence of a mass-radius relation naturally leads to such a form as described by Eqs. (2) and (3) because these clusters still generate stars, so logically those with smaller populations must simply be younger.

Equation (3) probably directly describes the star formation process in leaky clusters since loss processes, such as escaping stars only play a minor role. Weidner et al. (2011) show that in the embedded phase only about $15-20 \%$ of the cluster mass is lost through escapers with little consequence to the cluster radius. If a direct relation between cluster radius and cluster age exists, Eq. (3) implies strongly accelerated star formation.

\subsection{Star formation history}

What does the embedded cluster sequence tell us about the star formation in a cluster environment? Two star formation scenarios would be possible: i) either stars only form in the central high-density area, or ii) the star formation process itself progresses from the inside outwards. We assume that the cluster size is a function of time of the form $r_{\mathrm{cl}} \propto t^{c}$, where $c$ is a constant with $0 \leq c \leq 1$. Limiting our investigation to $c<1$ means excluding accelerated cluster expansion. Equation (3) translates into $M_{\mathrm{c}} \propto t^{1.7 c}$. In case i) the velocities would have to be supervirial so that as time progresses the cluster size expands. This could possibly be caused by early gas expansion. However, this would require all clusters to have the same velocity dispersion.

Case ii) is easier to reconcile with these findings. In a homogenoeus medium, one would expect $M_{\mathrm{c}} \propto t^{3}$ if star formation just proceeded linearly from the centre outwards. Since the observations indicate an $M_{\mathrm{c}} \propto t^{1.7 c}$-dependence, this would either require the star formation front to decelerate with time $r_{\mathrm{cl}} \propto t^{0.6 c}$ or the medium to become less dense. The latter fits what we expect from both theory and observations, where the gas density in the cluster forming clumps decreases from the centre outwards.

The gas density $\rho_{\mathrm{g}}=M(r) / V$ in young forming clusters (Larson 1981; Chandrasekhar 1939) is proportional either to $r^{-1}$ or $r^{-2}$. Besides, power-law density profiles with slopes in the range 1.5-2.0 are put forward for molecular clumps by various studies (see Parmentier 2011, and references therein). This means that the matter available for star formation has the same dependency. In the first case one would end up with the gas mass increasing as $M_{\mathrm{c}} \propto r^{2}$, and the second would result in a dependency $M_{\mathrm{c}} \propto r^{1}$. Assuming a constant star formation efficiency throughout the cluster and using the size-age relation, this corresponds to the cluster mass increasing as $M_{\mathrm{c}} \propto t^{2 c}$ and $M_{\mathrm{c}} \propto t^{c}$, respectively. Generally, one could conclude that for a linear cluster expansion $(c=1)$, the gas density distribution in the clump from which these clusters form would have to have the form $M_{\mathrm{c}} \propto r^{1.3}$.

An alternative picture would be that it is not the density distribution that is responsible for the shape of the observed density-age relation, but the way that star formation proceeds. Here it is assumed that star formation continues in the central area while the star formation front moves outwards in a medium with an $r^{-2}$ gas density dependence. This is a likely scenario, because star formation would probably not immediately stop in the centre but continue until enough massive stars have formed to blow a cavity into the gas in the central area. For simplicity a model assuming linearly propagating star formation has been calculated in Fig. 3 (dashed line). In this picture in each shell of thickness $\Delta r$ initially the same amount of star formation $\Delta M$ happens, but the further a shell is from the centre, the later it starts to produce stars. After its onset the star formation rate slows down by the factor $\alpha_{r}$ in each of the individual shells as the gas content in these regions decreases. Assuming that the star formation proceeds over $N$ shells, the total star formation $M_{\mathrm{SF}}$ is given by

$$
M_{\mathrm{SF}}(N)=\sum_{i=i}^{N} \sum_{t=1}^{N} \alpha_{r}^{t-i} \Delta M=\sum_{i=i}^{N}(N-i+1) \alpha_{r}^{N-i+1} \Delta M .
$$

This model fits well with the derived density radius relation shown in Fig. 3 if we choose $\alpha_{r}\left(t_{n+1}\right)=0.7 * \alpha_{r}\left(t_{n}\right)$.

These simple estimates should be regarded as a first attempt to show how new insights into embedded cluster dynamics might provide clues to the star formation process itself. This should motivate future observations, as well as more comprehensive numerical modelling. 


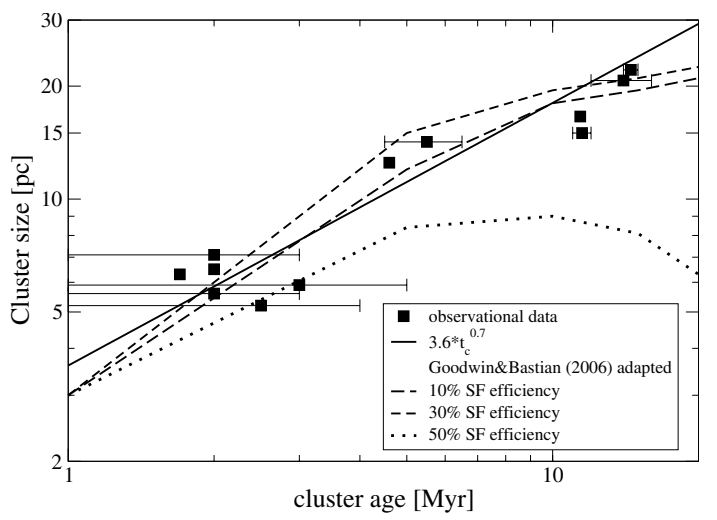

Fig. 4. Cluster size development as a function of cluster age for exposed clusters. The observational values were taken from Wolff et al. (2007), and simulation data points are from Goodwin \& Bastian (2006). A linear fit in the log-log space is shown as drawn line, scaled versions of the results of Goodwin \& Bastian (2006) for instantaneous gas expulsion with $10 \%$ (short dashed line), 30\% (long dashed line), and 50\% (dotted line) star formation (SF) efficiency are shown.

\subsection{Gas expulsion}

As Fig. 2 demonstrates, there are two distinct phases in the density development of leaky clusters: the star formation phase, which leads to a density/radius relation as expressed by Eq. (2), and the cluster expansion phase described by Eq. (1). We stress that while the sequence of leaky clusters stems from dynamical evolution (i.e. post-gas expulsion expansion), the embedded cluster track is the imprint of the formation process. The "switch" from one to the other process is the gas expulsion as illustrated by Fig. 3 .

Since the cluster expansion and mass loss are the result of the star formation process and the subsequent gas expulsion, it should be possible to draw conclusions about the early history of these clusters from the properties of these two processes. Using results from existing numerical simulations, we can place constraints on how the gas expulsion is likely to proceed in leaky clusters.

Goodwin \& Bastian (2006) simulate a situation similar to that in leaky clusters. Their cluster simulations contained 15000 stars distributed according to a Plummer model with a core radius of $1 \mathrm{pc}$. They investigated the process of instantaneous gas expulsion by assuming virial equilibrium before gas expulsion and varied the star formation (SF) efficiency. Here SF efficiency is defined as $M_{\text {stars }} /\left(M_{\text {stars }}+M_{\text {gas }}\right)$ with $M_{\text {stars }}$ being the mass of the stars in a cluster and $M_{\text {gas }}$ the gas mass. Leaky clusters have a somewhat larger number of stars, typically $N \sim 20000-40000$.

Figure 4 shows the radial development of leaky clusters as a function of cluster age for the data used in Pfalzner (2009). Here the drawn line represents Eq. (1), which is basically a linear fit in the log-log space. We note the relatively large errors of 1-2 Myr in cluster age. In addition, Fig. 4 shows the simulation results of Goodwin \& Bastian (2006) scaled to the initial cluster parameters relevant here. Such a scaling is possible since the model excludes scattering processes between the cluster stars. It can be seen that a low SF efficiency of $10 \%$ leads to somewhat smaller cluster radii than observed, whereas a high SF efficiency of $50 \%$ would only lead to an increase in cluster radii by a factor of $\sim 2$. The best fit is obtained by an instantaneous gas expulsion with $30 \%$ SF efficiency. In this case the simulated and observed leaky cluster expansion are consistent. The data of the $30 \%$ SF efficiency simulation actually seem a better fit to the observational data than Eq. (1). However, the age determination has such a large error that it is not possible to decide which one is more realistic. Only better observational data and simulations tailored to the actual parameters of leaky clusters will give an answer to this question.

It has been suggested that the distinction between starburst and leaky clusters is basically identical to that of those systems that remain bound and becoming unbound after gas expulsion (Portegies Zwart et al. 2010; Gieles \& Portegies Zwart 2011) and that the leaky clusters are therefore (unbound) associations. However, taking the SF efficiency of 30\% derived above, the results of Goodwin \& Bastian (2006) indicate that despite the considerable mass loss of these clusters, about $10 \%$ to $15 \%$ of the stars can be expected to remain bound and develop into a cluster that lasts $>100 \mathrm{Myr}$. Although much reduced in mass, these bound entities still consist of $\sim 1000$ stars, albeit with a low volume density of the order of $1 M_{\odot} \mathrm{pc}^{-3}$. It is a matter of cluster definition, if such a group of stars is still called a cluster. If one regards boundedness of a sufficient number of stars as the prime property of a cluster then it is. In contrast, if one requires a certain volume or surface density (for a review of the different definitions see Bressert et al. 2010) then it is a cluster at the start of the expansion, but it can no longer regarded as such at later stages. In either case more detailed investigations should be carried out to specifiy the properties of these remaining entities more precisely.

\section{Conclusion}

In this paper we investigated the formation and early development of leaky clusters. This first approach is far from definitive, but it does provide a consistent picture. We showed that the difference between leaky and starburst clusters is not related to mass but must lie in their early development (see Fig. 1). Taking our cue from the size/age relation demonstrated for young leaky exposed clusters, we postulated an analogous relation for the embedded phase. The following conclusions are based on this concept.

- We examined the star formation history of leaky clusters by determining the cluster radius development as opposed to the traditional examination of the stellar age distribution in single clusters. Here the global properties of a multitude of known embedded clusters were used to make deductions about the star formation history inside individual star clusters. This is only possible because recent findings strongly suggest that there is a common development for leaky clusters.

- The radial cluster development implies that star formation initially proceeds at a low rate and accelerates considerably later on. Spatially this is consistent with star formation in a sphere of increasing volume.

- The increase in cluster mass with cluster size follows a dependence of the form $M_{\mathrm{c}}=a r_{\mathrm{cl}}^{b}$ with $a=359 \pm 15$ and $b=1.71 \pm 0.07$, and the cluster density develops as $\rho \approx 100 r_{\mathrm{cl}}^{-1.3}$.

- It has been shown that the observational results of cluster expansion are consistent with a $30 \%$ star formation efficiency followed by an instantaneous gas expulsion.

From the existing data it is not absolutely clear whether the star formation process is the result of two distinct generations of stars or a continuous but accelerated star formation. From the massradius diagram (Fig. 3) the latter seems more likely. 
With these results a picture of the mass and density development of leaky clusters can be constructed over the first $20 \mathrm{Myr}$ of their existence. Leaky clusters start out with several tens of stars being confined within $\sim 0.2-0.3 \mathrm{pc}$, then grow in mass and cluster membership as $M_{\mathrm{c}} \sim 360 r_{\mathrm{cl}}^{1.7}$ until they reach their maximum mass of several $10^{4} M_{\odot}$ with a size of $\sim 3-5 \mathrm{pc}$. Then gas expulsion sets in and the clusters expand rapidly while losing most of their mass over the next 20 Myr. Throughout the cluster formation phase the density (at least in the central region) is so high that the cluster environment might influence the planet formation process significantly.

In this paper the focus was on the early evolution of leaky clusters, it would be highly desirable to obtain the equivalent information for starburst clusters. However, at present it is impossible to determine in which way star formation in starburst clusters differs from that in leaky clusters. Figure 1 implies that the precursors of starburst clusters would form an extension of the starburst cluster sequence towards smaller radii $(<0.1 \mathrm{pc})$ at density of $>10^{5-7} M_{\odot} \mathrm{pc}^{-3}$. Otherwise it would require them to contract and increase in density while simultanuously forming a high number of massive stars. No massive embedded cluster has been definitely classified as a starburst precursor so far possibly with the exception of W43 (Motte et al. 2003; Nguyên Lu'O'Ng et al. 2011) This is not surprising because starburst clusters are situated either close to the Galactic centre $(8 \mathrm{kpc})$ and/or along spiral arms. At these locations the higher background density makes cluster detection much more difficult, so that even most of the exposed massive starburst clusters have only been detected recently.

Acknowledgements. I wish to thank A. Stolte, C. Olczak, G. Parmentier, and M. Gieles for very helpful discussions.

\section{References}

Adams, F. C., Proszkow, E. M., Fatuzzo, M., \& Myers, P. C. 2006, ApJ, 641, 504 Baraffe, I., Chabrier, G., \& Gallardo, J. 2009, ApJ, 702, L27

Bastian, N., Gieles, M., Goodwin, S. P., et al. 2008, MNRAS, 389, 223 Baumgardt, H., \& Kroupa, P. 2007, MNRAS, 380, 1589

Borissova, J., Ivanov, V. D., Hanson, M. M., et al. 2008, A\&A, 488, 151

Bressert, E., Bastian, N., Gutermuth, R., et al. 2010, MNRAS, 409, L54

Carpenter, J. M., Heyer, M. H., \& Snell, R. L. 2000, ApJS, 130, 381

Chandrasekhar, S. 1939, An introduction to the study of stellar structure (The University of Chicago press)

Comerón, F., Pasquali, A., Figueras, F., \& Torra, J. 2008, A\&A, 486, 453

Figer, D. F. 2008, in Massive Stars as Cosmic Engines, Proc. IAU Symp., 250, 247

Gieles, M., \& Portegies Zwart, S. F. 2011, MNRAS, 410, L6

Goodwin, S. P., \& Bastian, N. 2006, MNRAS, 373, 752

Haşegan, M., Jordán, A., \& Côté, P. 2005, ApJ, 627, 203

Herbst, W., Herbst, D. K., Grossman, E. J., \& Weinstein, D. 1994, AJ, 108, 1906 Hills, J. G. 1980, ApJ, 235, 987

Küpper, A. H. W., Maschberger, T., Kroupa, P., \& Baumgardt, H. 2011, MNRAS, 1397

Lada, C. J., \& Lada, E. A. 2003, ARA\&A, 41, 57

Larson, R. B. 1981, MNRAS, 194, 809

Motte, F., Schilke, P., \& Lis, D. C. 2003, ApJ, 582, 277

Murray, N. 2009, ApJ, 691, 946

Nguyên Lu'O’Ng, Q., Motte, F., \& Schuller, F. 2011, A\&A, 529, A41

Palla, F., \& Stahler, S. W. 2000, ApJ, 540, 255

Parmentier, G. 2011, MNRAS, 413, 1899

Pfalzner, S. 2009, A\&A, 498, L37

Portegies Zwart, S. F., McMillan, S. L. W., \& Gieles, M. 2010, ARA\&A, 48, 431 Preibisch, T., \& Zinnecker, H. 1999, AJ, 117, 2381

Pudritz, R. E. 2002, Science, 295, 68

Swift, J. J., \& Williams, J. P. 2008, ApJ, 679, 552

Wolff, S. C., Strom, S. E., Dror, D., \& Venn, K. 2007, AJ, 133, 1092

Weidner, C., Bonnell, I. A., \& Moeckel, N. 2011, MNRAS, 410, 1861

Wright, N. J., Drake, J. J., Drew, J. E., \& Vink, J. S. 2010, ApJ, 713, 871 